\title{
Fatigue Reliability Analysis for the Mechanical System of Large-scale Medical Imaging Equipment SPECT
}

\author{
Lifeng Guo ${ }^{1, a,{ }^{*}}$, Ruijing Zhang ${ }^{1, b}$, Liguo Liü ${ }^{1, c}$, Hui Gao ${ }^{1, d}$ and Jianping Xue \\ ${ }^{1}$ School of Mechanical Engineering, Beijing Institute of Petrochemical Technology, Beijing, China \\ 2Beijing Novel Medical Equipment Ltd., Beijing, China \\ aguolifeng@bipt.edu.cn, b13662086915@163.com, c1550195537@qq.com, ${ }^{\mathrm{d}}$ gaohui@bipt.edu.cn, \\ exuejianping@novelmedical.cn \\ ${ }^{*}$ Corresponding author
}

Keywords: SPECT, dynamic simulation, finite element analysis, fatigue reliability analysis.

\begin{abstract}
The mechanical system of large-scale medical imaging equipment SPECT is complex and expensive, so it is difficult to carry out the reliability test with a large number of physical equipments. The virtual test method is used to evaluate the reliability of the system. The virtual prototype of SPECT mechanical system is established with SOLIDWORKS. The force load between components was obtained through dynamic simulation in ADAMS software. Finite element static analysis in ANSYS software was used to obtain the stress and strain distribution. Based on the S-N fatigue analysis method, the fatigue life cloud chart and the fatigue damage cloud chart of the key components are calculated in the nCode software to determine the fatigue failure site, which provides the basis for the reliability design and equipment maintenance of the SPECT mechanical system.
\end{abstract}

\section{大型医学影像设备SPECT机械系统疲劳可靠性分析}

\author{
郭丽峰 $1, a,{ }^{*}$, 张瑞静 ${ }^{1, b}$, 刘立国 ${ }^{1, c}$, 高辉 $1, d$, 薛建平 $2, e$ \\ 1 北京石油化工学院机械工程学院, 北京, 中国 \\ 2北京永新医疗设备有限公司, 北京, 中国
}

aguolifeng@bipt.edu.cn, b13662086915@163.com, c1550195537@qq.com, ${ }^{\mathrm{d}}$ gaohui@bipt.edu.cn, exuejianping@novelmedical.cn

*通讯作者

关键词: SPECT; 动力学仿真; 有限元分析; 疲劳可靠性

中文摘要. 针对大型医学影像设备SPECT的机械系统结构复杂、造价昂贵, 很难用大量物理 设备进行可靠性试验问题，采用可靠性虚拟试验方法对其进行可靠性分析评估。应用 SOLIDWORKS软件建立SPECT机械系统虚拟样机; 在ADAMS软件中进行动力学仿真分析, 得到零部件之间的作用力载荷; 在ANSYS软件中进行有限元静力学分析, 得到应力、应变分 布; 基于 S-N疲劳分析法, 在nCode软件中计算得到关键零部件的疲劳寿命云图和疲劳损伤云 图，确定易疲劳失效部位，为SPECT机械系统的可靠性设计及设备维护提供依据。 


\section{1. 引言}

大型医学影像设备SPECT (Single Photon Emission Computed Tomography) 在成像过程中, 要求其机械系统驱动两个重达数百公斤的探测器环绕人体运动, 以满足不同成像模式需求。 机械系统的故障将直接导致设备停机甚至威胁人生安全。医疗器械安全标准要求该类设备关 键部件的安全系数不低于 $4[1]$ 。即便如此, 近年在SPECT设备临床使用过程中, 仍发生了由 于机械故障导致探测器掉落, 并将患者压死的重大医疗事故[2]。

SPECT机械系统结构复杂, 造价昂贵, 很难用大量物理设备开展可靠性评估试验。随着 虚拟样机技术和可靠性技术的发展, 虚拟疲劳试验方法日趋成熟, 在汽车、船舶、飞机等领 域有广泛应用[3-6]。本文在对SPECT机械系统工作模式分析基础上, 采用ADAMS软件进行动 力学仿真分析, 应用ANSYS 软件进行有限元静力学分析, 进而在疲劳分析软件nCode中应用 S-N法对SPECT机械系统关键零部件进行疲劳可靠性分析, 以期保证SPECT设备结构设计的可 靠性、安全性。

\section{SPECT机械系统}

SPECT机械系统主要由机架、主回转运动机构、探测器平移及旋转机构组成, 如图1所示。 机架固定不动, 是SPECT设备的载体。主回转机构可绕Z轴进行 $360^{\circ}$ 旋转运动。探测器平移 及旋转机构位于主回转运动机构部件上, 驱动探测器沿X1(或X2)、Y1(或Y2)向做线性平移运 动和绕Z1(或Z2)轴做旋转运动。位于传动机构末端的探测器质量达400 500 Kg。

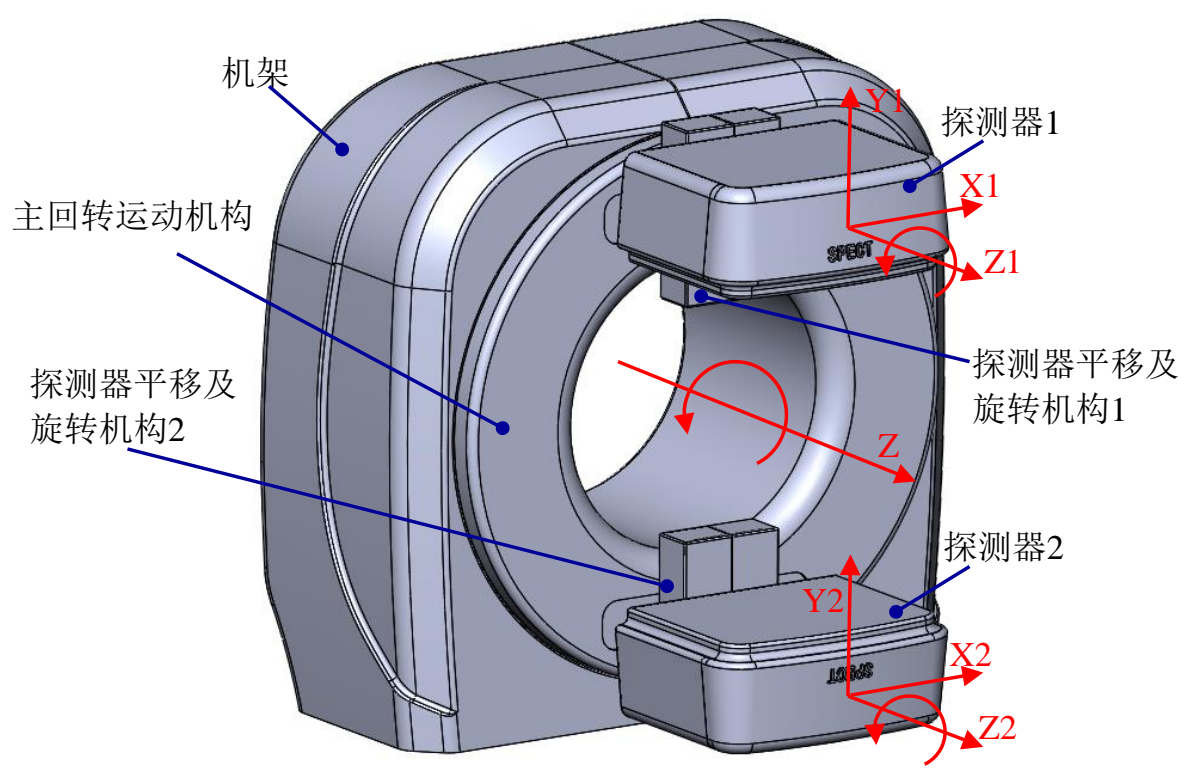

图1 SPECT机械系统组成及运动自由度

当主回转运动机构 $360^{\circ}$ 转动时, 各零部件将承受周期性变化载荷。因此, 为保证SPECT 设备运行安全, 不仅要求零部件及其连接结构满足医疗器械要求的强度要求标准 (4 8倍安全 系数), 还应具有可靠性的靠疲劳能力和足够的疲劳寿命。SPECT结构复杂, 零部件数量达 数百, 本文以与设备安全密切相关的X向丝杜轴承座组件为例阐述㾉劳可靠性分析过程。

\section{3. 动力学仿真}

动力学仿真的目的是得到SPECT在各种运动模式下零部件之间的作用载荷。这些作用力 将作为疲劳载荷用于零部件的疲劳寿命分析中。经过简化和载荷等效处理后的SPECT机械系 统虚拟样机如图2所示。将SPECT模型导入动力学仿真软件ADAMS中, 根据零部件连接和运 动情况进行机构建模。SPECT机械系统中典型运动副定义如图3所示。 


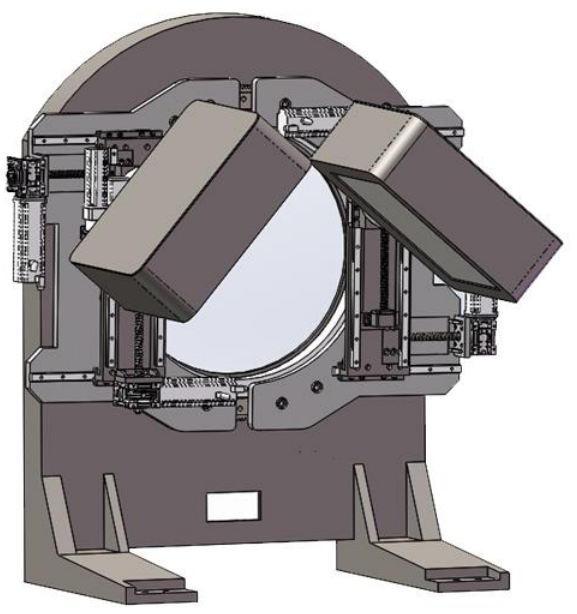

图2 SPECT机械系统虚拟样机
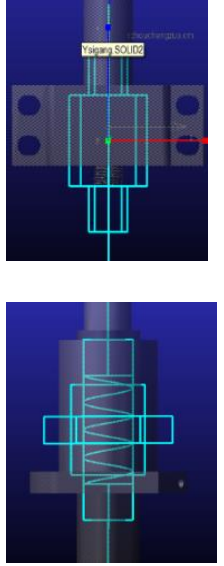

图3 典型运动副定义

根据SPECT设备工况, 在各运动副施加驱动, 即可获得各运动副以及各零部件之间的作 用力。以图 2 所示 $180^{\circ}$ 成像模式为例, 在主回转机构的主回转齿轮上施加 $n=2 \mathrm{r} / \mathrm{min}$ 的旋转驱 动, 可获得 $\mathrm{X}$ 向丝杜与轴承座之间的轴向接触力如图4所示。将所获得接触力以 dac格式文件导 出并保存，为后续疲劳分析提供载荷谱。

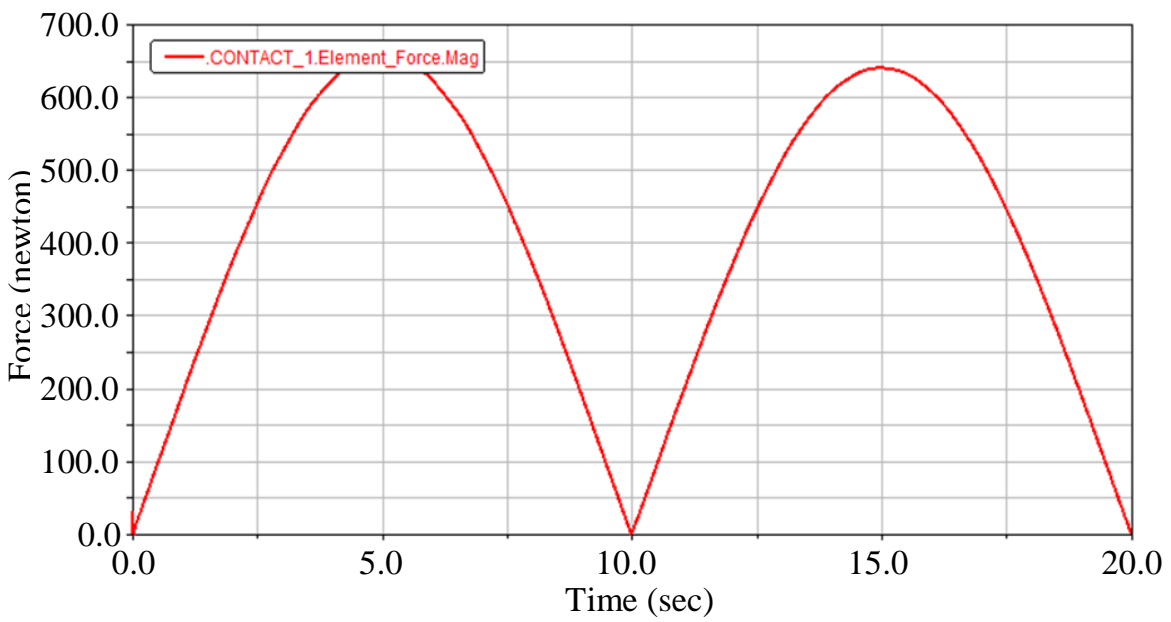

图4 X向丝杜与轴承座之间的轴向接触力

\section{4. 有限元静力学分析}

通过有限元静力学分析获得单位载荷作用下零部件应力分布, 为后续疲劳分析中提供应 力谱数据。在ANSYS软件中完成网格划分的X向丝杜轴承座组件如图5所示。

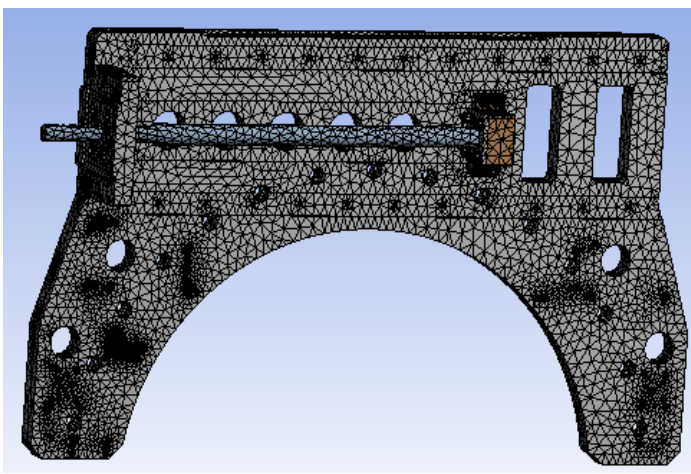

图5 X向丝杜轴承座组件有限元网格划分

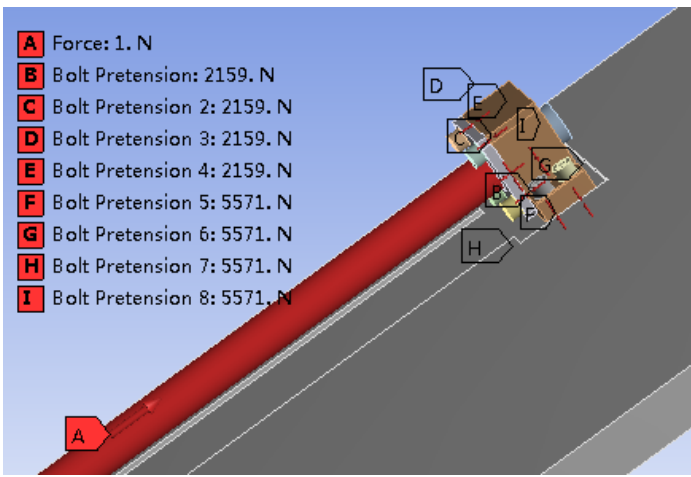

图6 X向丝杜轴承座组件载荷设置 
轴承座材料为经调制处理的 45 号钢, 屈服强度 $490 \mathrm{MPa}$, 最大抗拉强度 $700 \mathrm{MPa}$; 螺钉为 12.9 级高强螺钉，材料为调制处理铬钼合金钢，屈服强度 $1000 \mathrm{MPa}$, 最大抗拉强度 1200 $\mathrm{MPa}$ 。在底板与与主回转连接的螺钉孔处, 施加固定约束。对轴承座固定螺钉施加预紧力, 沿丝杜轴线对丝杜施加单位载荷。施加边界条件的X向丝杜轴承座组件如图6所示。经ANSYS Workbench求解得到的X向丝杜轴承座组件应力、变形分布云图分别如图7、图8所示。

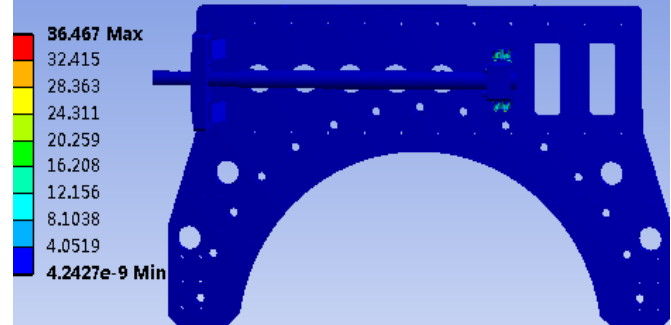

图7 X向丝杜轴承座组件有限元网格划分

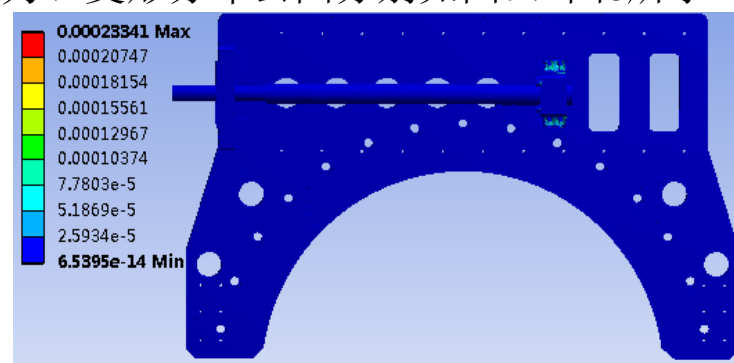

图8Ｘ向丝杜轴承座组件载荷设置

\section{5. 疲劳可靠性分析}

在nCode软件中加载ADAMS动力学仿真结果 (*.dac文件) 和ANSYS有限元计算结果 (*.rst 文件），采用名义应力法（S-N法）进行疲劳可靠性分析，分析界面如图9所示。根据轴承座 和螺钉的材料特性，在 $\mathrm{nCode}$ 中选择适当的 S-N曲线。经可靠性虚拟疲劳分析，测得X向丝杜 轴承座和螺钉的寿命云图及损伤云图分别如图10至图13所示。

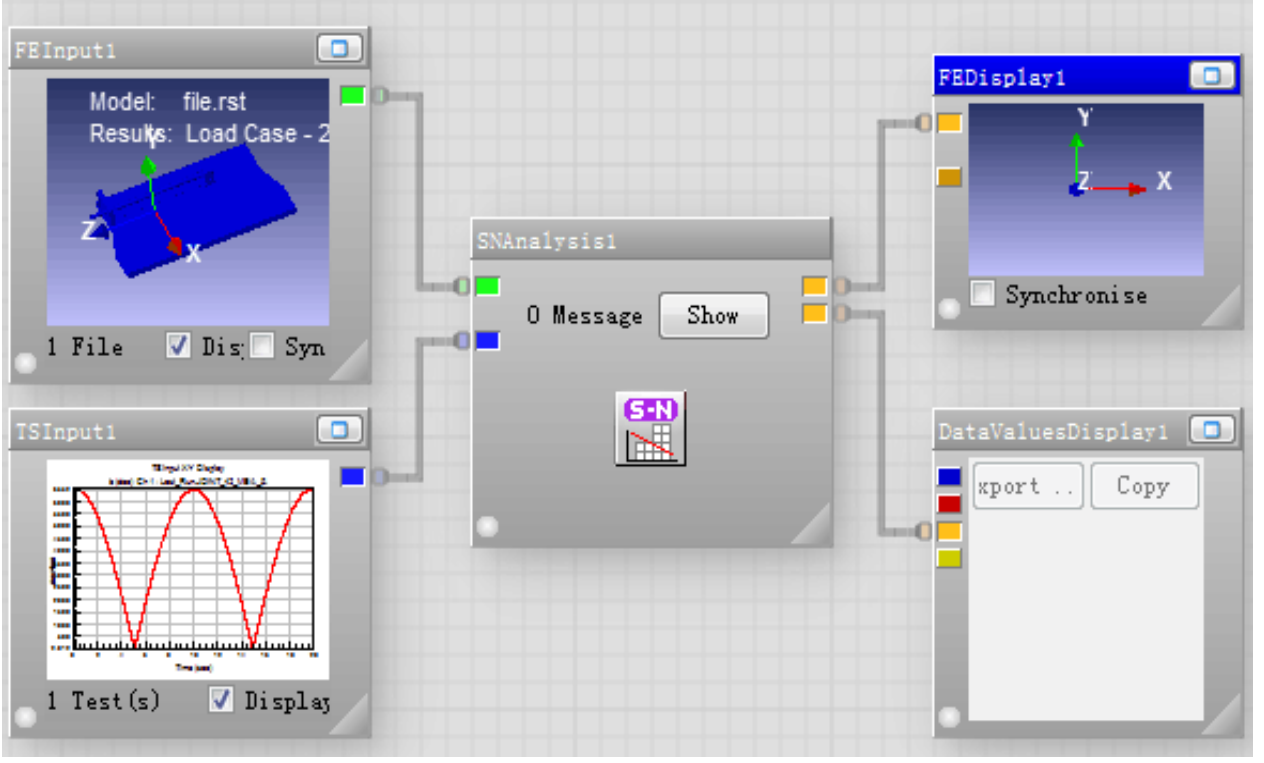

图9＼cjkstart疲劳可靠性分析流程图

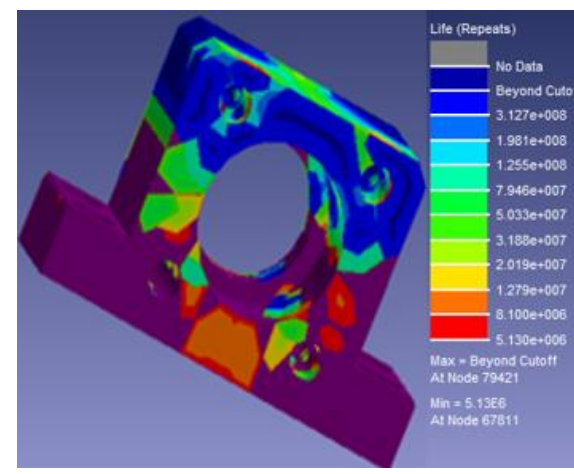

图10 轴承座疲劳寿命分布云图

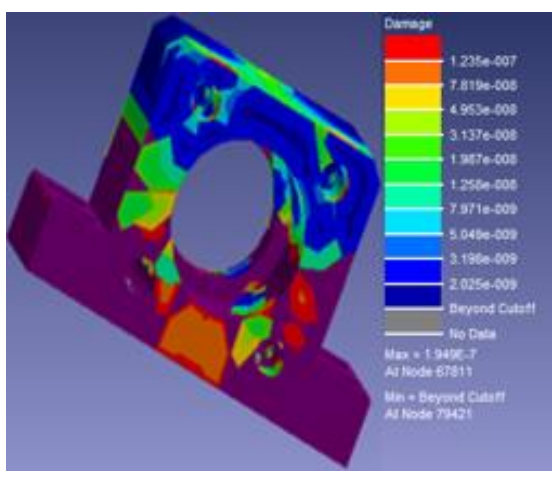

图11 轴承座疲劳损伤分布云图 


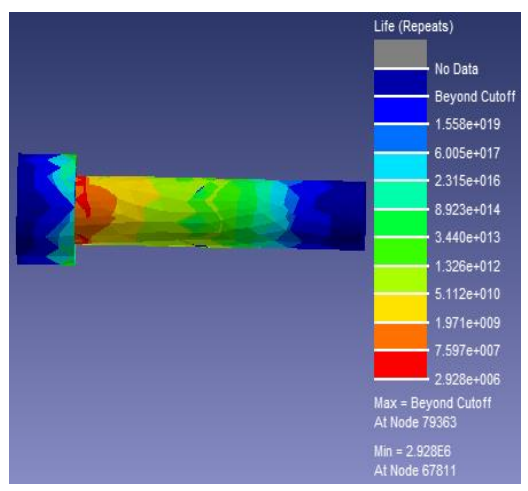

图12 螺钉疲劳寿命分布云图

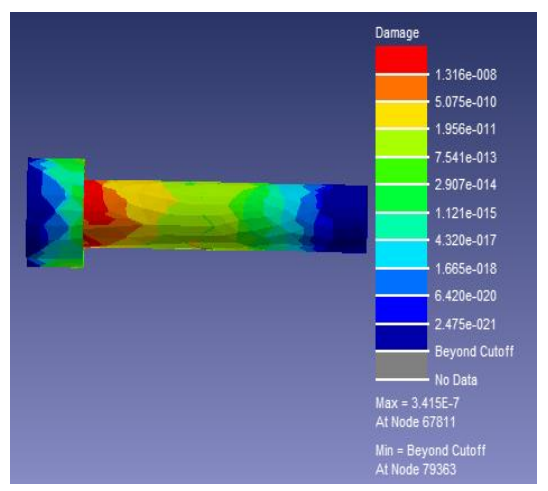

图13 螺钉疲劳损伤分布云图

从疲劳寿命图中可以得到：轴承座的寿命最小为5.13E6次循环，根据实际工况换算的时 间寿命为 53.4 年; 螺钉的寿命最小为 $2.928 \mathrm{E} 6$ 次循环，根据实际工况换算的时间寿命为 30.5 年。 这两个部件的寿命均满足整机工作寿命 15 年的设计指标。从疲劳损伤云图可以得到：轴承座 最早发生疲劳失效的位置位于轴承座底部中间位置, 螺钉最早发生疲劳失效的位置位于螺钉 杆与螺钉头连接部位。

\section{6. 结束语}

在对SPECT机械系统虚拟样机工作模式分析的基础上, 通过多体动力学软件ADAMS、有 限元分析软件ANSYS Workbench和疲劳分析软件nCode的联合仿真, 获得X向轴承座组件的疲 劳寿命和疲劳损伤分布结果。这为相关零部件的优化设计、后期维护, 以及运行状态监测提 供了理论依据。

\section{致谢}

本文为北京市教育委员会市属高校创新能力提升计划项目 “大型医学影像设备SPECT机 电系统研制”（编号: TJSHG201310017035）的阶段性成果之一。

\section{References}

[1] Medical electrical equipment Part 1: General requirements for safety, GB9706.1-2007/IEC 60601-1:1988, pp.44, 2007.

[2] Dave Fornell, Patient's death from falling scanner component spurs major recall, September 13, 2013. https://www.itnonline.com/article/patient-killed-during-nuclear-imaging-scan.

[3] Wei, Zhigang, Zhu, Xuebing, Chen, Xiaohua, et al. Fatigue life analysis of rear bushing of automobile control arm on bench test, Journal of Mechanical Engineering, vol. 52, pp. 81-89, 2016.

[4] Li Chenglin, Song Shasha, Han Zhennan, Fatigue Reliability Analysis of Frame Based on nCode Design-Life, JOURNAL OF GRAPHICS, vol. 35, pp. 42-45, 2014.

[5] SUN Xiaoqing, SHI Duanwei, Dynamics simulation and fatigue life prediction of drive system of vertical shiplift, Engineering Journal of Wuhan University, vol. 49, pp. 610-615, 2016.

[6] JIANG Chengjie, XU Feng, The fatigue life analysis and optimization of landing gear for a large amphibian aircraft, Machine Design and Manufacturing Engineering, vol. 44, pp. 22-28, 2015 . 